\title{
Association between cytosolic expression of BRCA1 and metastatic risk in breast cancer
}

\author{
W L Santivasi ${ }^{1}$, H Wang ${ }^{2}$, T Wang $^{3}$, Q Yang ${ }^{4}$, X Mo ${ }^{5}$, E Brogi ${ }^{6}$, B G Haffty ${ }^{4}$, A B Chakravarthy ${ }^{3}$ and Fen Xia*,1 \\ ${ }^{1}$ Department of Radiation Oncology, The Ohio State University College of Medicine, 072 A Starling Loving Hall, 300 W 10 th \\ Avenue, Columbus, OH 43212, USA; '2 Department of Lung Cancer, The 307 Hospital of the People's Liberation Army, 8 East Street, \\ Fengtai, Beijing, People's Republic of China; ${ }^{3}$ Department of Radiation Oncology, Vanderbilt University School of Medicine, \\ B1003 Preston Research Building, 1301 22nd Avenue South, Nashville, TN 37232, USA; ${ }^{4}$ Department of Radiation Oncology, \\ Robert Wood Johnson Medical School, 195 Little Albany Street, New Brunswick, NJ 08901, USA; ${ }^{5}$ Center for Biostatistics, \\ The Ohio State University, 2012 Kenny Road, Room 244, Columbus, OH 43210, USA and ${ }^{6}$ Department of Pathology, Memorial \\ Sloan Kettering Cancer Center, 1275 York Avenue, New York, NY 10021, USA
}

Background: Although BRCA1 has been extensively studied for its role as a tumour-suppressor protein, the role of BRCA1 subcellular localisation in oncogenesis and tumour progression has remained unclear. This study explores the impact of BRCA1 mislocalisation on clinical outcomes in breast cancer.

Methods: Tissue microarrays assembled from a cohort of patients with all stages of breast cancer were analysed for BRCA1 localisation and correlated with patient survival. Tissue microarrays of patients who had breast cancer that had metastasised to the lung were assembled from an independent cohort of patients. These were analysed for BRCA1 subcellular expression. In vitro studies using cultured human breast cancer cells were conducted to examine the effect of cytosolic BRCA1 on cell migration and efficiency of invasion.

Results: An inverse association was found between cytosolic BRCA1 expression and metastasis-free survival in patients aged $>40$ years. Further analysis of BRCA1 subcellular expression in a cohort of breast cancer patients with metastatic disease revealed that the cytosolic BRCA1 content of breast tumours that had metastasised to the lung was $36.0 \%(95 \% \mathrm{Cl}=(31.7 \%, 40.3 \%)$, which was markedly higher than what is reported in the literature (8.2-14.8\%). Intriguingly, these lung metastases and their corresponding primary breast tumours demonstrated similarly high cytosolic BRCA1 distributions in both paired and unpaired analyses. Finally, in vitro studies using human breast cancer cells demonstrated that genetically induced BRCA1 cytosolic sequestration (achieved using the cytosol-sequestering BRCA1 5382insC mutation) increased cell invasion efficiency.

Conclusions: Results from this study suggest a model where BRCA1 cytosolic mislocalisation promotes breast cancer metastasis, making it a potential biomarker of metastatic disease.

BRCA1 is a genome guardian and tumour suppressor that demonstrates a number of diverse cellular functions depending on its subcellular localisation. BRCA1 is a nuclear-cytoplasmic shuttling protein. When localised in the nucleus, it functions in cell proliferation, DNA damage response and repair, and transcriptional regulation (Yang and Xia, 2010). When localised in the cytoplasm, BRCA1 triggers apoptosis via a p53-independent mechanism in human breast cancer cells (Rodriguez et al, 2004; Rakha et al, 2008; Jiang et al, 2011). BRCA1 also interacts with acetyl-CoA carboxylase, which is required for breast cancer cell survival and is associated with cancer progression (Magnard et al, 2002; Chajes et al, 2006; Moreau et al, 2006; Fang et al, 2014). Export of BRCA1 to the cytoplasm is dependent on an importin/ CRM1 (chromosomal region maintenance 1)-mediated nuclear/

*Correspondence: Dr F Xia; E-mail: fen.xia@osumc.edu

Received 25 March 2015; revised 5 May 2015; accepted 13 May 2015; published online 9 June 2015

(c) 2015 Cancer Research UK. All rights reserved 0007-0920/15 
cytoplasmic shuttling system regulated by a number of tumoursuppressor proteins, including p53 and BARD1 (BRCA1-associated RING domain protein 1) (Feng et al, 2004; Henderson, 2005; Thompson, 2010; Jiang et al, 2011).

Although it is well established that germline mutations of BRCA1 are associated with familial breast and ovarian cancers, there is increasing evidence that mutations which affect BRCA1's nuclear/ cytoplasmic shuttling and its resultant cytosolic accumulation are also associated with breast cancer (Rodriguez et al, 2004). Specifically, cancer-associated mutations of the C-terminal BRCT (BRCA1 carboxyl-terminal) domain of BRCA1 restrict the protein to the cytosol and diminish its nuclear function in homologous recombinational repair of DNA breaks (Rodriguez and Henderson, 2000; Fabbro et al, 2004; Rodriguez et al, 2004; Li et al, 2008; Jiang et al, 2011).

It is known that germline BRCA1-mutated familial breast and ovarian cancers demonstrate an increased incidence of metastasis, with brain and lung being the most common metastatic sites (Stoppa-Lyonnet et al, 2000; Antoniou et al, 2003; Albiges et al, 2005). Moreover, BRCA1 cytosol accumulation has been shown to independently predict survival, tumour grade, and recurrence in low-grade basal-like breast cancers (Rakha et al, 2008). However, the role of BRCA1 in sporadic breast cancer, including its subcellular localisation and its clinical relevance, remain controversial (Taylor et al, 1998; Lee et al, 1999; Yoshikawa et al, 1999; Yang et al, 2001, 2002). In contrast to the well-established function of BRCA1 in the nucleus where it regulates DNA damage response and repair pathways, little is known about the functional role of BRCA1 when it accumulates in the cytoplasm and how cytosolic mislocalisation of BRCA1 affects breast cancer initiation and progression (Henderson, 2012).

To investigate the effects of cytoplasmic accumulation of BRCA1 on breast cancer progression, we first evaluated the relationship between BRCA1 subcellular expression and clinical outcomes of breast cancer patients. Second, we assessed the relationship between BRCA1 subcellular location and metastasis to the lung using tissue microarrays of patients with breast cancer for whom we also had tissue available both from their primary tumour as well as from their lung metastases. Finally, we examined in vitro supporting evidence that genetic induction of BRCA1 cytosolic retention in human breast cancer cells enhanced cell invasiveness.

\section{MATERIALS AND METHODS}

\section{Patient data and tissue microarrays}

Assembly of tissue microarrays of the primary breast tumours and corresponding lung metastases. The study protocols were reviewed and approved by the institutional review board of their corresponding institutions (Yale Cancer Center and MemorialSloan Kettering Cancer Center (MSKCC)). To investigate the relationship between BRCA1 subcellular expression and clinical outcomes of breast cancer patients, formalin-fixed paraffinembedded tissue blocks containing invasive breast carcinomas and normal breast tissue were retrieved, along with the corresponding H\&E-stained slides, from the archives of the Yale University School of Medicine. To ensure uniformity of sectioning, older paraffin blocks were melted and re-embedded using modernday plastic cassettes. Areas of invasive carcinoma, distinct from in situ components and normal tissue elements, were identified and marked for subsequent retrieval and analysis. Core biopsies of $0.6 \mathrm{~mm}$ in diameter were taken from each donor block and arrayed into a recipient paraffin block $(45 \mathrm{~mm} \times 20 \mathrm{~mm})$ using a tissue puncher/arrayer as described (Kononen et al, 1998). Patients selected for this study were treated at Yale between 1970 and 2005. Of this group of 504 patients, 406 cases with evaluable tumour cores were stained for BRCA1 distribution.
To study the relationship between BRCA1 subcellular location and metastasis to the lung, tissue blocks containing metastatic lung tumours, as well as their corresponding primary breast tumours were retrieved, along with the corresponding H\&E-stained slides, from the archives of Department of Pathology at MSKCC. Fifty-four tissue specimens of primary breast carcinomas and 80 lung metastases were obtained from a total of 106 patients treated at MSKCC. Of these, only 41 primary breast specimens also had paired lung metastasis specimens $(n=77)$ and contained evaluable tumour cores that were stained for BRCA1 distribution. Patients selected for the study were treated at MSKCC between 1986 and 2006.

Measures of patient outcomes. Overall survival was defined as the time from initial diagnosis to the time of death. Loco-regional recurrence-free survival was defined as the time from initial diagnosis to the time of relapse in the ipsilateral breast and/or regional nodes according to clinical and/or radiographic evidence. Distant metastasis-free survival (MFS) was defined as the time from initial diagnosis to the time of occurrence of distant disease according to clinical and/or radiographic evidence.

Immunofluorescence assay. Five- $\mu$ m-thick sections of formalinfixed, paraffin-embedded tissue microarrays were de-paraffinised and rehydrated. Samples were pretreated to allow antigen retrieval with Target Retrieval Solution (DAKO, Carpinteria, CA, USA). Sections were blocked with $3 \%$ hydrogen peroxide, followed by blocking in $2 \%$ goat serum/0.1\% Triton-X 100/PBS ( $1 \mathrm{~h})$. Slides were then incubated with a mouse monoclonal primary antibody against the amino-terminal 304 amino acids of human BRCA1 (Ab-1, Catalog Number OP92, Calbiochem, Dermstadt, Germany). This antibody was used at a dilution of $1: 50$, with overnight incubation at $4{ }^{\circ} \mathrm{C}$. Several studies have confirmed the reliability and reproducibility of this antibody when used in formalin-fixed materials (Taylor et al, 1998; Yoshikawa et al, 1999; Perez-Valles et al, 2001). Slides were washed with PBS and incubated with secondary antibody (1:1000 goat anti-mouse Alexa594-conjugated antibodies, Molecular Probes, Thermo Fisher Scientific, Carlsbad, CA, USA), stained with DAPI for nuclear visualisation for 1-2 min, and analysed by fluorescence microscopy (Carl Zeiss, Thornwood, NY, USA). Both cytoplasmic and nuclear BRCA1 immunoreactivity were revealed by the BRCA1 Ab-1 antibody. The specificity of immunostaining with BRCA1 antibody was confirmed by the absence of staining by preincubation of the antibody with excess of the peptide immunogen. Negative controls had the primary antibody omitted and replaced by non-immune normal serum from the same species as the primary antibody. BRCA1 subcellular expression was scored separately by two independent researchers who were blinded to patient outcomes. The overall staining intensity in cancer cells was scored on a 0 to +3 scale, as previously described: $0=$ no staining, $1=$ weak-to-moderate staining, 2 = moderate staining, and $3=$ moderate-to-strong staining (Wang et al, 2013). A wide range of quantification criteria have been reported to classify BRCA1 expression patterns as nuclear or cytosolic (Rakha et al, 2008; Wang et al, 2013; Mylona et al, 2014). For the purposes of this study, cases where $>60 \%$ of tumour cells had moderate-to-strong nuclear immune detection of BRCA1 were considered as positive nuclear expression tumours $(n=316)$. Cases where $>15 \%$ of tumour cells had moderate-to-strong cytosolic immune detection of BRCA1 were considered as positive cytosolic expression tumours $(n=54)$. Tumours with BRCA1 expression falling below these levels were not eligible and excluded for the correlative analysis $(n=36)$.

\section{Statistical analysis}

Analyses used to examine BRCA1 expression and clinical outcomes. These analyses were conducted on the primary breast tumour tissue microarrays assembled from the Yale Cancer Center 
patient cohort. The association of cytosolic BRCA1 expression with overall survival, loco-regional recurrence-free survival, and distant MFS was analysed initially by log-rank test and further with Cox regression to adjust for other prognostic factors in breast cancers. The type I error rate was set to 0.05. Data analysis was conducted with SAS 9.3 (SAS Inc., Cary, NC, USA).

Analyses used to examine BRCA1 expression and metastasis. These analyses were conducted using the lung metastasis tissue microarrays assembled from the MSKCC patient cohort. The comparisons of the percentages of cells demonstrating cytosolic BRCA1 expression between breast primary tumours and their corresponding lung metastases were conducted by two-sample $t$-test for unpaired samples or paired $t$-test for matched samples. Type I error rate was set to 0.05 . Data analysis was conducted with SAS 9.3 (SAS Inc., Cary, NC, USA).

In vitro invasion and migration assays. $\mathrm{MCF}-7$ cells expressing wild-type BRCA1 or mutant BRCA1/5382insC were seeded onto the top chamber of Transwell filters $(8-\mu \mathrm{m}$ pore) (VWR Scientific, Radnor, PA, USA) coated with fibronectin. The filters were placed in a 24-well plate. Recombinant soluble vascular endothelial growth factor receptor 2 (VEGFR2 (ExFlk)) in 1\% BSA was added to a final concentration of $10 \mathrm{ng} \mathrm{ml}^{-1}$ in the lower chamber as a chemoattractant. MCF-7 cells were counted to assess their migration (eight fields per filter) after $5 \mathrm{~h}$ of incubation.

Invasion assays were carried out using Matrigel precoated inserts (BD Biosciences, San Jose, CA, USA) following the manufacturer's instructions. VEGF was placed in the lower wells. The wild-type BRCA1- or mutant BRCA1/5382insC-expressing MCF-7 cells were loaded into each of the upper wells. The chambers were incubated for $18-20 \mathrm{~h}$ at $37^{\circ} \mathrm{C}$. Following incubation, the inserts were removed, and the non-invading cells on the upper surface were removed with a Q-tip. The cells on the lower surface of the membrane were counted in eight high-power fields (HPF) under a light microscope (Carl Zeiss). The invasion efficiency of cells was calculated using the formula: invasion efficiency $=($ mean invasion $/$ mean migration $) \times 100 \%$.

\section{RESULTS}

Patient outcomes, tissue microarrays, and BRCA1 subcellular expression. Tissue and clinical data from breast cancer patients with or without metastases were collected at Yale Cancer Center and MSKCC. Table 1 summarises the clinical and pathological characteristics of the patients treated at Yale. This tissue microarray was used for the analysis as detailed in the 'Cytosolic BRCA1 expression and clinical outcomes' section. These specimens were primary breast tumours from patients with all stages of breast cancer. Table 2 summarises the clinical and pathological characteristics of the patients treated at MSKCC, and the specimens of the metastatic lung metastatic tumours and corresponding primary breast tumours were assembled in microarrays. These tissue microarrays were used for the analyses as detailed in the 'Cytosolic BRCA1 expression and lung metastasis in breast cancer patients' section. These specimens were lung metastatic tumours and breast primary tumours from patients with metastatic breast cancer.

To determine the subcellular expression of BRCA1 in tumour cells, tissue microarrays were analysed by immunofluorescent staining of BRCA1 protein. BRCA1 expression by tumour cells was categorised based on BRCA1 staining pattern: nuclear or cytosolic (Feng et al, 2004; Wang et al, 2010). Representative nuclear and cytosolic BRCA1 staining is shown in Figure 1A. Negative control experiments using a competing peptide to block the anti-BRCA1 antibody (Figure 1B), as well as rabbit IgG in place of the anti-
Table 1. Clinicopathological characteristics of Yale tumour tissue samples

\begin{tabular}{|c|c|c|}
\hline Characteristics & Number & $\%$ \\
\hline \multicolumn{3}{|l|}{ Age } \\
\hline$>40$ years & 405 & 80 \\
\hline$\leqslant 0$ years & 99 & 20 \\
\hline \multicolumn{3}{|l|}{ Tumour size } \\
\hline$\leqslant 2 \mathrm{~cm}$ & 352 & 70 \\
\hline$>2 \mathrm{~cm}$ & 103 & 20 \\
\hline Unknown & 49 & 10 \\
\hline \multicolumn{3}{|c|}{ Lymph node status } \\
\hline Negative & 233 & 46 \\
\hline Positive & 82 & 16 \\
\hline Unknown & 189 & 38 \\
\hline \multicolumn{3}{|l|}{ ER status } \\
\hline Negative & 213 & 42 \\
\hline Positive & 262 & 52 \\
\hline Unknown & 29 & 6 \\
\hline \multicolumn{3}{|l|}{ PR status } \\
\hline Negative & 236 & 47 \\
\hline Positive & 241 & 48 \\
\hline Unknown & 27 & 5 \\
\hline \multicolumn{3}{|l|}{ Her2 status } \\
\hline Negative & 400 & 79 \\
\hline Positive & 80 & 16 \\
\hline Unknown & 24 & 5 \\
\hline \multicolumn{3}{|l|}{ Triple negative } \\
\hline No & 333 & 66 \\
\hline Yes & 141 & 28 \\
\hline Unknown & 30 & 6 \\
\hline \multicolumn{3}{|c|}{ Adjuvant hormone therapy } \\
\hline No & 311 & 62 \\
\hline Yes & 191 & 38 \\
\hline Unknown & 2 & 0 \\
\hline \multicolumn{3}{|c|}{ Adjuvant radiation therapy } \\
\hline No & 0 & 0 \\
\hline Yes & 504 & 100 \\
\hline \multicolumn{3}{|c|}{ BRCA1 distribution } \\
\hline Nuclear & 316 & 63 \\
\hline Nuclear-cytosolic & 134 & 27 \\
\hline Cytosolic & 54 & 11 \\
\hline
\end{tabular}

BRCA1 antibody are shown in Figures $1 \mathrm{~B}$ and $\mathrm{C}$, respectively. In this study, a tumour with nuclear BRCA1 expression was defined by $>60 \%$ of cells exhibiting positive BRCA1 staining only in the nuclei. Cytosolic expression was defined by $>15 \%$ of cells exhibiting positive BRCA1 staining only in the cytosolic compartment.

Cytosolic BRCA1 expression and clinical outcomes. We first sought to investigate whether cytosolic expression of BRCA1 was associated with a clinically significant difference in patient prognosis in a cohort of patients diagnosed with early and late stage breast cancers treated at Yale Cancer Center. Table 1 summarises the clinical and pathological characteristics of the Yale patients from whom these samples were taken. These were patients with primary breast cancers with or without metastatic disease. Analysis of the BRCA1 subcellular distribution in these tissue microarrays demonstrated no significant differences in overall survival $(P=0.85)$ or loco-regional recurrence-free survival $(P=0.25)$ between patients who had high cytosolic BRCA1 expression $(n=54)$ in the primary breast tumours $v s$ those with a nuclear pattern $(n=316)$ (Kaplan-Meier curves not shown). Interestingly, this comparison revealed a trend towards decreased MFS $(P=0.06)$ (Figure 2A). Thirty-six cases demonstrated BRCA1 
distribution in both the nucleus and cytosol and were not included in this analysis. Multivariate analysis with adjustment for other known prognostic factors, including tumour size, tumour grade, treatment, lymph node status, ER status, PR status, Her2 status, and use of systemic therapy, revealed that only age had a statistically significant association $(P=0.03)$.

Sporadic breast cancer patients aged $>40$ years rarely carry germline BRCA1 mutations, and BRCA1 could be compromised by deficiency in the regulation of its function, such as BRCA1 shuttling and subsequent mislocalisation (Antoniou et al, 2003). We next performed an analysis specifically within patients aged $>40$ years $(n=318)$. This analysis included 36 cases with cytosolic BRCA1 expression and 257 with nuclear expression. Twenty-five cases that demonstrated BRCA1 distribution in both the nucleus and cytosol were not included in this analysis. This comparison demonstrated a significant association between cytosolic BRCA1 breast tumours and decreased MFS $(P=0.04$; Figure 2B). Our

\begin{tabular}{|c|c|c|}
\hline Subcategory & Number & $\%$ \\
\hline \multicolumn{3}{|l|}{ Tumour size } \\
\hline$\leqslant 2 \mathrm{~cm}$ & 35 & 33 \\
\hline$>2 \mathrm{~cm}$ & 37 & 35 \\
\hline Unknown & 34 & 32 \\
\hline \multicolumn{3}{|c|}{ Lymph node status } \\
\hline Negative & 25 & 24 \\
\hline Positive & 68 & 64 \\
\hline Unknown & 13 & 12 \\
\hline \multicolumn{3}{|l|}{ ER status } \\
\hline Negative & 20 & 19 \\
\hline Positive & 28 & 26 \\
\hline Unknown & 58 & 55 \\
\hline \multicolumn{3}{|l|}{ PR status } \\
\hline Negative & 29 & 27 \\
\hline Positive & 20 & 19 \\
\hline Unknown & 57 & 54 \\
\hline \multicolumn{3}{|l|}{ Her2 status } \\
\hline Negative & 41 & 39 \\
\hline Positive & 8 & 8 \\
\hline Unknown & 57 & 54 \\
\hline
\end{tabular}

A

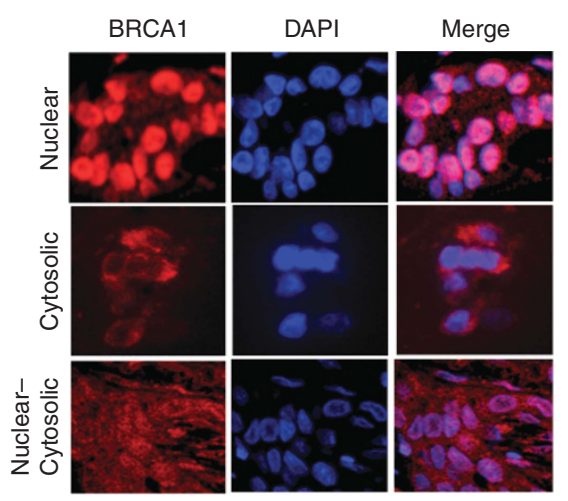

analysis did not demonstrate a significant relationship between BRCA1 location and the rate of loco-regional tumour recurrence, suggesting that loco-regional recurrence may not significantly contribute to the observed decrease in MFS in this study. Furthermore, these observations suggest a potential contribution of dysfunction mediated by mislocalisation of BRCA1 to breast cancer metastasis.

Cytosolic BRCA1 expression and lung metastasis in breast cancer patients. To further investigate the potential relationship underlying the observed decreased MFS in tumours with cytosolic BRCA1 expression, we examined BRCA1 subcellular expression in lung metastases from breast cancer. This cohort of breast cancer patients with lung metastases was selected from an independent population diagnosed with metastatic breast cancers and treated at MSKCC. Table 2 summarises the clinical and pathological characteristics of the MSKCC patients from whom these samples were taken. We observed that the tumours that had metastasised to the lung exhibited a high level of cytosolic expression of BRCA1 $(\bar{x}=36.0 \%, 95 \% \mathrm{CI}=(31.7 \%, 40.3 \%))$. This is in contrast to previous reports that BRCA1 is predominantly a nuclear protein, with only $8.2-14.8 \%$ of primary breast tumours demonstrating a BRCA1 cytosolic distribution pattern (Fabbro et al, 2004; Kennecke et al, 2010).

We then examined the BRCA1 expression patterns in primary breast tumours that gave rise to those lung metastases. Using the same techniques, we examined BRCA1 subcellular distribution in the primary breast tumours from patients with lung metastases in the same MSKCC cohort. Intriguingly, the proportion of cells showing cytosolic expression in these 41 primary breast tumours was as high as the 77 lung metastatic tumours $(38.5 \pm 10.5 \%$ vs $36.0 \pm 18.9 \% ; P=0.36$ ) (Figure 3A). A further comparison, which paired 18 of these metastatic lung tumours with their corresponding primary breast tumours, also found no significant difference in cytosolic BRCA1 expression (35.8 $\pm 19.2 \%$ vs $37.8 \pm 12.3 \%$; $P=0.69$ ) (Figure 3B). This similarly high level of cytoplasmic BRCA1 expression in both the lung metastatic tumours and their corresponding primary breast tumours suggests that BRCA1 cytosolic expression may be an early event, acting as one of the causative factors, rather than a late event in the development of metastasis.

In vitro cytosolic BRCA1 sequestration, cell migration, and cell invasion. To explore a potential mechanism underlying cytosolic

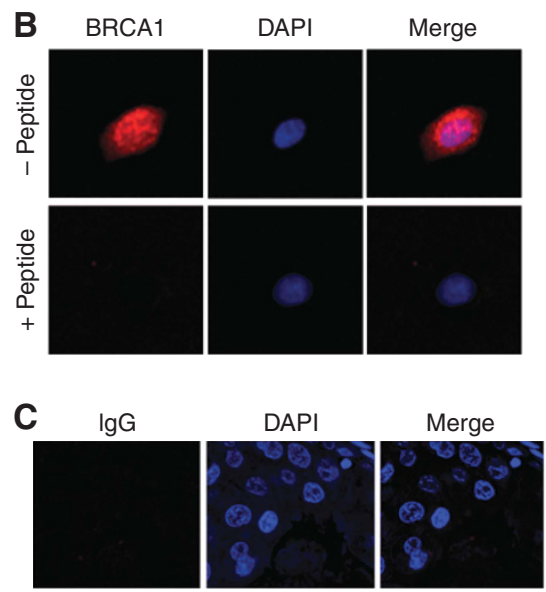

Figure 1. Immunofluorescence staining to determine BRCA1 subcellular expression. (A) Representative immunofluorescence staining of nuclear, cytosolic, and nuclear-cytosolic BRCA1 expression in breast tumour cells. Nuclear and cytosolic categorisations were counted in the immunofluorescence assay, while nuclear-cytosolic samples were excluded from analyses. (B) Demonstration of immunospecificity of the BRCA1 antibody. Immunospecificity was verified by a lack of staining when the antibody was preincubated with excess of the peptide immunogen. (C) Negative control of BRCA1 antibody staining. This incubation omitted the primary antibody, with replacement of the primary antibody by non-immune normal serum from the same species (rabbit). 


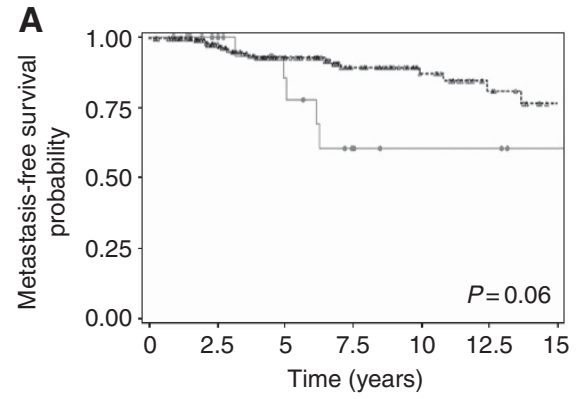

-AA-Ar. Nuclear BRCA1

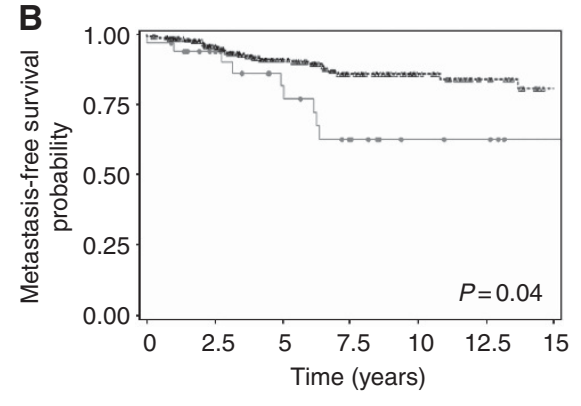

- Cytosolic BRCA1

Figure 2. MFS as a function of BRCA1 subcellular expression in breast cancer patients. (A) MFS of patients with nuclear ( $n=316$ ) vs cytosolic $(n=54)$ BRCA1 distribution in primary breast tumours (log-rank test, $P=0.06$ ). This analysis used the primary breast tumour tissue microarray assembled from the Yale patient cohort. A Cox regression was used to adjust for age, tumour size, tumour grade, treatment, and lymph node, ER, PR, and Her2 statuses. (B) MFS of patients aged $>40$ years with nuclear $(n=257)$ vs cytosolic $(n=36)$ BRCA1 distribution in primary breast tumours (log-rank test, $P=0.04$ ). This analysis used the primary breast tumour tissue microarray assembled from the Yale patient cohort. A Cox regression was used to adjust for age, tumour size, tumour grade, treatment, and lymph node, ER, PR, and Her2 statuses.
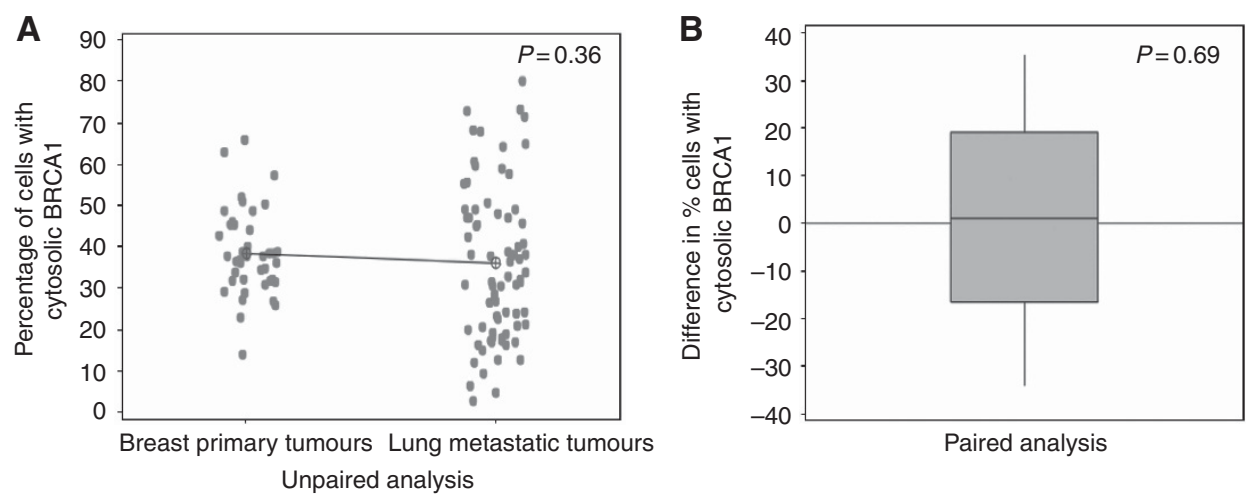

Figure 3. Comparison of cytosolic BRCA1 distribution in lung metastatic tumours and primary breast tumours. (A) Cytosolic BRCA1 proportion in breast primary tumours $(n=41 ; \bar{x}=38.5 \pm 10.5 \% ; P=0.36)$ vs lung metastatic tumours $(n=77 ; \bar{x}=36.0 \pm 18.9 \%)$. This analysis used the metastatic lung tumour and the breast primary tissue to assemble microarrays from the MSKCC patient cohort. Each dot represents a sample, and the means of each tumour type are connected by a line. (B) Paired comparison of cytosolic BRCA1 proportion in lung metastases vs their corresponding breast primary tumours. The difference between the paired samples was not significant $(n=18 ; 95 \% \mathrm{Cl}(-8.2 \%, 12.15 \%) ; P=0.69)$.

location of BRCA1 in tumour cell metastasis, we examined whether a genetic predisposition towards BRCA1 cytosolic sequestration could alter tumour cells' metastatic capacity in vitro. It has been shown previously that the metastatic potential of a cancer cell depends on both migration and invasion and that these are two different processes regulated by distinct mechanisms (Scully et al, 1997). In our in vitro migration and invasion assays, cells that expressed either wild-type BRCA1 or mutant BRCA1 5382insC, a common mutation in germline breast cancers, which predominantly localises BRCA1 protein to the cytoplasm, were used (Rodriguez et al, 2004; Antoniou et al, 2005). This sequestration occurs via loss of BRCT domain-mediated nuclear localisation (Huyton et al, 2000; Antoniou et al, 2003).

This analysis demonstrated significantly increased invasion in MCF-7 cells expressing 5382insC mutant BRCA1 (located predominantly in the cytosol) compared with those expressing wild-type BRCA1 (located predominantly in the nucleus) (4.8vs 1.7 cells $/ \mathrm{HPF}, P=0.03$; Figure $4 \mathrm{~A}$ ). This occurred despite the fact that BRCA1 5382insC mutant-expressing MCF-7 cells exhibited decreased migration compared with those expressing wild-type BRCA1 (95.1 vs 136.8 cells/HPF, $P<0.0001$; Figure 4B). Invasion was normalised to account for migration by the formula: invasion efficiency $=($ mean invasion $/$ mean migration $) \times 100 \%$. The 5382 insC mutants demonstrated a four-fold increase in invasion efficiency (5.04\% vs $1.24 \%, P=0.03$; Figure $4 \mathrm{C}$ ). This finding indicates that the cytosolic sequestration of BRCA1 can increase breast tumour cell invasiveness. Together with the observed decreased MFS in patients with cytosolic BRCA1 breast cancers and the dramatically higher level of BRCA1 cytosolic expression in breast cancer patients whose tumour metastasised to the lung, our data suggest a potential role of cytosolic BRCA1 expression in promoting breast cancer metastasis.

\section{DISCUSSION}

In this study, we observed a potential association of cytosolic BRCA1 expression with metastasis in breast cancer using tissue microarray immunofluorescence analysis and in vitro invasion assays. Our analyses demonstrated that cytosolic BRCA1 is associated with decreased MFS, especially in patients aged $>40$ years. Our studies also revealed that BRCA1 is highly concentrated in the cytosol of both the primary as well as the tumours that have metastasised to the lung. Finally, our in vitro results suggest that genetically induced sequestration of BRCA1 in the cytosol increases cell invasiveness. Together, our data indicate a potential association between BRCA1 cytosolic expression and risk of metastatic development.

In the past, there had been debate regarding BRCA1's cytoplasmic location (Chen et al, 1995; Scully et al, 1997). 

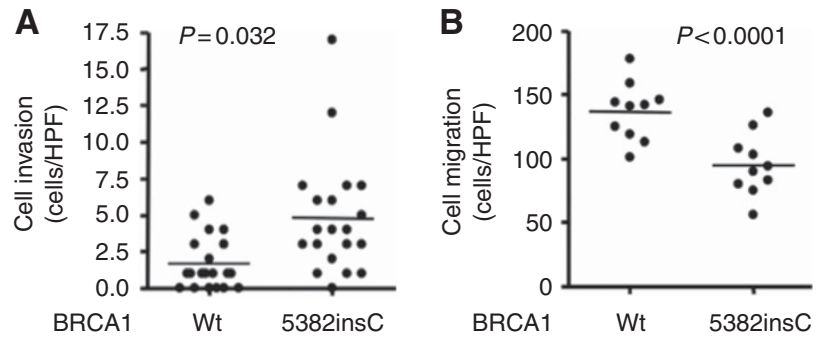

\begin{tabular}{|c|c|c|c|}
\hline BRCA1 & $\begin{array}{c}\text { Mean } \\
\text { invasion }\end{array}$ & $\begin{array}{c}\text { Mean } \\
\text { migration }\end{array}$ & $\begin{array}{c}\text { Invasion } \\
\text { efficiency }\end{array}$ \\
\hline $\mathrm{Wt}$ & 1.7 & 136.8 & $1.24 \%$ \\
\hline 5382 insC & 4.8 & 95.1 & $5.04 \%$ \\
\hline
\end{tabular}

Figure 4. Invasion and migration of cultured human breast cancer MCF7 cells with genetically modulated BRCA1 subcellular distribution. (A) Invasion of cells expressing wild-type BRCA1 or cytosolic mutant BRCA1 (5382insC) (4.8 vs 1.7 cells/HPF, $P=0.03$ ). VEGF was used as a chemoattractant, and migratory cells were counted after 18-20 h of incubation. (B) Migration of cells expressing wild-type BRCA1 or cytosolic mutant BRCA1 (5382insC) (95.1 vs 136.8 cells/HPF, $P<0.0001)$. VEGFR2 was used as a chemoattractant, and invading cells were counted after $5 \mathrm{~h}$ of incubation. (C) Normalised mean invasion efficiency of MCF7 cells expressing wild-type BRCA1 or cytosolic mutant BRCA1 (5382insC) (5.04 vs $1.24 \%, P=0.03$ ). Invasion efficiency was calculated as the ratio of cell invasion (as represented in panel $\mathbf{A}$ ) to migration (as represented in panel B), expressed in percentage.

Although BRCA1 was initially identified to be predominantly located in the nucleus (Scully et al, 1997; De Potter et al, 1998; Coene et al, 2011), our results demonstrated that $38.5 \pm 10.5 \%$ of the cancer cells in both the primary tumours as well as its corresponding lung metastases (in the MSKCC patient cohort) showed cytosolic BRCA1 expression. We were able to demonstrate cytosolic BRCA1's association with decreased MFS. The lack of difference in loco-regional recurrence-free survival between patients with nuclear and cytosolic BRCA1 localisation suggests that the decrease in MFS is not secondary to local recurrence and is instead the result of the development of distant metastasis. These observations suggest a role of BRCA1 dysfunction in the development of metastasis. In support of our findings, previous studies have indicated that mislocalised BRCA1 in the cytosol serves as an independent predictor of survival, tumour grade, and recurrence in low-grade basal-like breast cancers (Rakha et al, 2008).

It has been well established that genomic instability, a hallmark of cancer, is one of the driving forces for any given tumour to give rise to a heterogeneous population of tumour cells and subclones with distinct biological features. As such, the observation that the high cytosolic enrichment of BRCA1 in lung metastatic tumours as well as in their primary breast tumours is intriguing and implies that acquisition of cytosolic expression of BRCA1 may predispose a subclone of cancer cells to metastasis, thus dictating the metastatic fate of the primary tumour. Our finding that genetic induction of BRCA1 sequestration to the cytosol is sufficient to alter breast cancer cell invasiveness strengthens this potentially causative relationship. Interestingly, previous studies have shown that BRCA1 is a regulator of caveolin and osteopontin, both of which promote tumour invasion and metastasis in vitro and in vivo (El-Tanani et al, 2006; Glait et al, 2006; Wang et al, 2008). Furthermore, it has been recently shown that BRCA1 is important for controlling spreading and motility via interacting and colocalising with ezrin/radixin/moesin and F-actin at the plasma membrane of breast cancer cells (Coene et al, 2011).

Our studies show that cytosolic BRCA1 is associated with a trend toward decreased MFS in all patients $(P=0.06)$. Interestingly, multivariate analysis with adjustment for other known prognostic factors revealed that only age had a statistically significant association $(P=0.03)$. This relationship becomes statistically significant in patients aged $>40$ years $(P=0.04)$. It is possible that this age-dependent relationship is a result of the relatively small size of our patient population $(n=370)$. It has been well documented that patients with germline mutations and dysfunctional BRCA1 tend to be diagnosed prior to age 40 years (Antoniou et al, 2003). On the other hand, BRCA1 mutations are rare in patients aged $>40$ years with sporadic breast cancer, and inactivation of BRCA1 function can occur through deregulation of BRCA1 subcellular expression. Interestingly, mutations that affect BRCA1's nuclear/cytoplasmic shuttling and its resultant cytosolic accumulation are also associated with breast cancer (Rodriguez et al, 2004). In the current study, we did not have the material available for determination of the BRCA1 mutation status of our patients. Future studies with larger sample sizes and genetic analysis of the BRCA1 gene will clarify whether this phenomenon holds true in all the age groups of breast cancer patients and the impact of BRCA1 mutation on this phenomenon.

In addition to a significant association between cytosolic BRCA1 and distant MFS, we have also seen that both the primary breast tumour and its corresponding lung metastasis demonstrate increased cytosolic BRCA1. Based on the findings of increased invasion efficiency demonstrated by cells with genetically induced cytosolic BRCA1 expression in our in vitro studies, we believe that these data support a model where cytosolic expression of BRCA1 predisposes cells to metastasis and promotes the metastatic risk of the primary breast tumour. Thus patients with high cytosolic BRCA1 in their tumours may have an increased risk of developing metastatic disease. Our data also suggest that BRCA1 mislocalisation may potentially serve as a biomarker of metastatic risk. Because of our relatively small sample size and limits on patient stratification, more in-depth analysis on a larger cohort of patients is needed to further understand the mechanisms involved in this novel role of cytosolic BRCA1 in cancer cell invasion and metastasis.

Although our study is suggestive of an association between cytosolic BRCA1 and breast cancer metastasis, additional investigation is warranted to further define the mechanisms by which cytosolic BRCA1 influences metastasis. Our results demonstrate a novel relationship between cytosolic BRCA1 subcellular expression and increased risk of developing metastasis. These findings may allow us to further our understanding of the mechanisms by which BRCA1 drives metastatic development and to use BRCA1 localisation as a potential biomarker for predicting risk of metastases and a potential therapeutic target in the treatment of breast cancer metastasis.

\section{ACKNOWLEDGEMENTS}

We gratefully acknowledge the technical support of Dr. Barbara Fingleton at Vanderbilt University in the completion of the in vitro studies. This work is supported by NIH grant CA118158, Susan G. Komen Breast Cancer Research Award BCTR0201704, and CTSA award UL1TR000445 from the National Center for Advancing Translational Sciences. Its contents are solely the responsibility of the authors and do not necessarily represent official views of the National Institutes of Health, Susan G. Komen Breast Cancer Foundation, or the National Center for Advancing Translational Sciences. 


\section{REFERENCES}

Albiges L, André F, Balleyguier C, Gomez-Abuin G, Chompret A, Delaloge S (2005) Spectrum of breast cancer metastasis in BRCA1 mutation carriers: highly increased incidence of brain metastases. Ann Oncol 16(11): 1846-1847.

Antoniou A, Pharoah PDP, Narod S, Risch HA, Eyfjord JE, Hopper JL, Loman N, Olsson H, Johannsson O, Borg A, Pasini B, Radice P, Manoukian S, Eccles DM, Tang N, Olah E, Anton-Culver H, Warner E, Lubinski J, Gronwald J, Gorski B, Tulinius H, Thorlacius S, Eerola H, Nevanlinna H, Syrjäkoski K, Kallioniemi O-P, Thompson D, Evans C, Peto J, Lalloo F, Evans DG, Easton DF (2003) Average risks of breast and ovarian cancer associated with BRCA1 or BRCA2 mutations detected in case series unselected for family history: a combined analysis of 22 studies. Am J Hum Genet 72(5): 1117-1130.

Antoniou AC, Pharoah PD, Narod S, Risch HA, Eyfjord JE, Hopper JL, Olsson H, Johannsson O, Borg A, Pasini B, Radice P, Manoukian S, Eccles DM, Tang N, Olah E, Anton-Culver H, Warner E, Lubinski J, Gronwald J, Gorski B, Tulinius H, Thorlacius S, Eerola H, Nevanlinna H, Syrjakoski K, Kallioniemi OP, Thompson D, Evans C, Peto J, Lalloo F, Evans DG, Easton DF (2005) Breast and ovarian cancer risks to carriers of the BRCA1 5382insC and 185delAG and BRCA2 6174delT mutations: a combined analysis of 22 population based studies. J Med Genet 42(7): 602-603.

Chajes V, Cambot M, Moreau K, Lenoir GM, Joulin V (2006) Acetyl-CoA carboxylase alpha is essential to breast cancer cell survival. Cancer Res 66(10): 5287-5294.

Chen Y, Chen CF, Riley DJ, Allred DC, Chen PL, Von Hoff D, Osborne CK, Lee WH (1995) Aberrant subcellular localization of BRCA1 in breast cancer. Science 270(5237): 789-791.

Coene ED, Gadelha C, White N, Malhas A, Thomas B, Shaw M, Vaux DJ (2011) A novel role for BRCA1 in regulating breast cancer cell spreading and motility. J Cell Biol 192(3): 497-512.

De Potter CR, Coene ED, Schelfhout VR (1998) Localization of BRCA1 protein at the cellular level. J Mammary Gland Biol Neoplasia 3(4): 423-429.

El-Tanani MK, Campbell FC, Crowe P, Erwin P, Harkin DP, Pharoah P, Ponder B, Rudland PS (2006) BRCA1 suppresses osteopontin-mediated breast cancer. J Biol Chem 281(36): 26587-26601.

Fabbro M, Schuechner S, Au WWY, Henderson BR (2004) BARD1 regulates BRCA1 apoptotic function by a mechanism involving nuclear retention. Exp Cell Res 298(2): 661-673.

Fang W, Cui H, Yu D, Chen Y, Wang J, Yu G (2014) Increased expression of phospho-acetyl-CoA carboxylase protein is an independent prognostic factor for human gastric cancer without lymph node metastasis. Med Oncol 31(7): 15 .

Feng Z, Kachnic L, Zhang J, Powell SN, Xia F (2004) DNA damage induces p53-dependent BRCA1 nuclear export. J Biol Chem 279(27): 28574-28584.

Glait C, Ravid D, Lee SW, Liscovitch M, Werner H (2006) Caveolin-1 controls BRCA1 gene expression and cellular localization in human breast cancer cells. FEBS Lett 580(22): 5268-5274.

Henderson BR (2005) Regulation of BRCA1, BRCA2 and BARD1 intracellular trafficking. Bioessays 27(9): 884-893.

Henderson BR (2012) The BRCA1 breast cancer suppressor: regulation of transport, dynamics, and function at multiple subcellular locations. Scientifica 2012: 796808 .

Huyton T, Bates PA, Zhang X, Sternberg MJ, Freemont PS (2000) The BRCA1 C-terminal domain: structure and function. Mutat Res 460(3-4): 319-332.

Jiang J, Yang ES, Jiang G, Nowsheen S, Wang H, Wang T, Wang Y, Billheimer D, Chakravarthy AB, Brown M, Haffty B, Xia F (2011) p53-Dependent BRCA1 nuclear export controls cellular susceptibility to DNA damage. Cancer Res 71(16): 5546-5557.

Kennecke H, Yerushalmi R, Woods R, Cheang MCU, Voduc D, Speers CH, Nielsen TO, Gelmon K (2010) Metastatic behavior of breast cancer subtypes. J Clin Oncol 28(20): 3271-3277.

Kononen J, Bubendorf L, Kallioniemi A, Barlund M, Schraml P, Leighton S, Torhorst J, Mihatsch MJ, Sauter G, Kallioniemi OP (1998) Tissue microarrays for high-throughput molecular profiling of tumor specimens. Nat Med 4(7): 844-847.

Lee WY, Jin YT, Chang TW, Lin PW, Su IJ (1999) Immunolocalization of BRCA1 protein in normal breast tissue and sporadic invasive ductal carcinomas: a correlation with other biological parameters. Histopathology 34(2): 106-112.

Li L, Wang H, Yang ES, Arteaga CL, Xia F (2008) Erlotinib attenuates homologous recombinational repair of chromosomal breaks in human breast cancer cells. Cancer Res 68(22): 9141-9146.

Magnard C, Bachelier R, Vincent A, Jaquinod M, Kieffer S, Lenoir GM, Venezia ND (2002) BRCA1 interacts with acetyl-CoA carboxylase through its tandem of BRCT domains. Oncogene 21(44): 6729-6739.

Moreau K, Dizin E, Ray H, Luquain C, Lefai E, Foufelle F, Billaud M, Lenoir GM, Venezia ND (2006) BRCA1 affects lipid synthesis through its interaction with acetyl-CoA carboxylase. J Biol Chem 281(6): 3172-3181.

Mylona E, Melissaris S, Nomikos A, Theohari I, Giannopoulou I, Tzelepis K, Nakopoulou L (2014) Effect of BRCA1 immunohistochemical localizations on prognosis of patients with sporadic breast carcinomas. Pathol Res Pract 210(8): 533-540.

Perez-Valles A, Martorell-Cebollada M, Nogueira-Vazquez E, Garcia-Garcia JA, Fuster-Diana E (2001) The usefulness of antibodies to the BRCA1 protein in detecting the mutated BRCA1 gene. An immunohistochemical study. J Clin Pathol 54(6): 476-480.

Rakha EA, El-Sheikh SE, Kandil MA, El-Sayed ME, Green AR, Ellis IO (2008) Expression of BRCA1 protein in breast cancer and its prognostic significance. Hum Pathol 39(6): 857-865.

Rodriguez JA, Au WW, Henderson BR (2004) Cytoplasmic mislocalization of BRCA1 caused by cancer-associated mutations in the BRCT domain. Exp Cell Res 293(1): 14-21.

Rodriguez JA, Henderson BR (2000) Identification of a functional nuclear export sequence in BRCA1. J Biol Chem 275(49): 38589-38596.

Scully R, Chen J, Ochs RL, Keegan K, Hoekstra M, Feunteun J, Livingston DM (1997) Dynamic changes of BRCA1 subnuclear location and phosphorylation state are initiated by DNA damage. Cell 90(3): 425-435.

Stoppa-Lyonnet D, Ansquer Y, Dreyfus H, Gautier C, Gauthier-Villars M, Bourstyn E, Clough KB, Magdelénat H, Pouillart P, Vincent-Salomon A, Fourquet A, Asselain B (2000) Familial invasive breast cancers: worse outcome related to BRCA1 mutations. J Clin Oncol 18(24): 4053-4059.

Taylor J, Lymboura M, Pace PE, A'Hern RP, Desai AJ, Shousha S, Coombes RC, Ali S (1998) An important role for BRCA1 in breast cancer progression is indicated by its loss in a large proportion of non-familial breast cancers. Int J Cancer 79(4): 334-342.

Thompson ME (2010) BRCA1 16 years later: nuclear import and export processes. FEBS Journal 277(15): 3072-3078.

Wang H, Yang ES, Jiang J, Nowsheen S, Xia F (2010) DNA damage-induced cytotoxicity is dissociated from BRCA1's DNA repair function but is dependent on its cytosolic accumulation. Cancer Res 70(15): 6258-6267.

Wang T, Wentz SC, Ausborn NL, Washington MK, Merchant N, Zhao Z, Shyr Y, Chakravarthy AB, Xia F (2013) Pattern of breast cancer susceptibility gene 1 expression is a potential prognostic biomarker in resectable pancreatic ductal adenocarcinoma. Pancreas 42(6): 977-982.

Wang Y, Yu J, Zhan Q (2008) BRCA1 regulates caveolin-1 expression and inhibits cell invasiveness. Biochem Biophys Res Commun 370(2): 201-206.

Yang ES, Xia F (2010) BRCA1 16 years later: DNA damage-induced BRCA1 shuttling. FEBS J 277(15): 3079-3085.

Yang Q, Sakurai T, Mori I, Yoshimura G, Nakamura M, Nakamura Y, Suzuma T, Tamaki T, Umemura T, Kakudo K (2001) Prognostic significance of BRCA1 expression in Japanese sporadic breast carcinomas. Cancer 92(1): 54-60.

Yang Q, Yoshimura G, Nakamura M, Nakamura Y, Suzuma T, Umemura T, Mori I, Sakurai T, Kakudo K (2002) BRCA1 in non-inherited breast carcinomas (Review). Oncol Rep 9(6): 1329-1333.

Yoshikawa K, Honda K, Inamoto T, Shinohara H, Yamauchi A, Suga K, Okuyama T, Shimada T, Kodama H, Noguchi S, Gazdar AF, Yamaoka Y, Takahashi R (1999) Reduction of BRCA1 protein expression in Japanese sporadic breast carcinomas and its frequent loss in BRCA1-associated cases. Clin Cancer Res 5(6): 1249-1261.

This work is published under the standard license to publish agreement. After 12 months the work will become freely available and the license terms will switch to a Creative Commons AttributionNonCommercial-Share Alike 4.0 Unported License 